\title{
Development and Validation of Novel Spectro- Chemometric, Chemometric and TLC-Densitometric Methods for Simultaneous Determination of Timolol and Travoprost in their Bulk Powders and Pharmaceutical Formulation
}

\author{
Hesham Salem*1,3, Afaf Aboulkheir ${ }^{2}$ and Basma E Abdel Aziz ${ }^{3}$ \\ ${ }^{1}$ Department of Pharmaceutical Chemistry, Faculty of Pharmacy, Deraya University, Egypt \\ ${ }^{2}$ Department of Analytical Chemistry, Faculty of Pharmacy, Zagazig University, Egypt \\ ${ }^{3}$ Faculty of Pharmacy, Deraya University, Egypt
}

*Corresponding author: Hesham Salem, Department of Pharmaceutical Chemistry, Faculty of Pharmacy, Deraya University, New Minia, Egypt.
Received Date: August 05, 2019

Published Date: August 27, 2019

\begin{abstract}
Simple, sensitive and reliable spectro-chemometric, Chemometric and thin layer chromatography (TLC)-densitometric methods were developed for simultaneous determination of binary mixture of timolol (TIM) and travoprost (TRA) without prior separation in their bulk drugs and dosage forms. The speectro-chemometric methods used are derivative spectrophotometry and isosbestic point methods. Multivariate methods involves the application of three chemometric techniques; classical least square (CLS) principal component regression (PCR) and partial least-squares regression (PLS) and thin-layer chromatography (TLC)-densitometric methods were developed for the simultaneous determination of TIM and TRA in presence of each other in bulk powder or in dosage form. Separation was carried out on merck HPTLC aluminium sheets of silica gel $60 \mathrm{~F} 254$ using ethyl acetate-methanol-ammonia (5:3:0.5) as mobile phase. All variables influencing the spectrophotometric and HPTLC methods were carefully investigated and optimized. The developed methods were evaluated according international conference of harmonization (ICH) rules. The current methods were successfully implemented for direct the determination of these drugs in their trade pharmaceutical formulations. The obtained results were contrasted with those calculated after application of reference method. The data obtained from both methods were statistically analyzed by Fand t-tests. The results of such study showed excellent agreement in respect to the accuracy and precision of the suggested and reference methods..
\end{abstract}

Keywords: Derivative spectrophotometry; Isosbestic point spectrophotometry; Multivariate chemometry

\section{Introduction}

Timolol (TIM) (Figure 1a) is (S)-1-(tert-butylamino)-3-[(4morpholin-4-yl-1,2,5-thiadiazole-3-yl) oxy] propan-2-ol. Timolol maleate is nonselective $\beta$-adrenergic receptors in the heart and decreases renin activity, all of which may play a role in reducing systolic and diastolic blood pressure. TIM is indicated for treatment of mild to moderate hypertension, reduction of mortality after myocardial infarction and migraine prophylaxis [1]. Travoprost (TRA) (Figure $1 \mathrm{~b}$ ) is chemically [1R-[1 $\alpha(\mathrm{Z}), 2 \beta(1 \mathrm{E}, 3 \mathrm{R}), 3 \alpha, 5 \alpha]]-$ 7-[3,5-Dihydroxy-2-[3-hydroxy-4[3-(trifluoromethyl) phenoxy]1-butenyl]cyclopentyl]-5-heptenoic acid-1-methyl-ethyleste. Travoprost is used in the treatment of glaucoma [1]. Both drugs are still used as a binary mixture in some pharmaceutical preparations for treatment of reduction of elevated intraocular pressure with open-angle glaucoma. Several analytical procedures are available for the analysis of the cited drugs in their bulk forms, multi-component mixtures, dosage forms or biological mixtures. These procedures are UV-Vis spectrophotometric [1-8], HPLC [9-18], HPTLC [1921] and Chemometric [22,23] assay methods are reported in the literature for the estimation of TIM and TRA individually and in combination with other dugs. For the time being only few reports were found on the direct determination of binary mixture of TIM and TRA through HPLC and direct UV methods [1,15-18]. For this reason, this study describes for the first time the simultaneous 
determination of TIM and TRA by spectro-chemometric such as first derivative (1D) and isosbestic point methods, multivariate methods involves the application of three chemometric techniques; classical least square (CLS) principal component regression (PCR) and partial least-squares regression (PLS) as well as thin-layer chromatography (TLC)-densitometric method, for simultaneous determination of the current drugs without prior separation in their authentic powder and available dosage form (Figure 1a \& 1b).
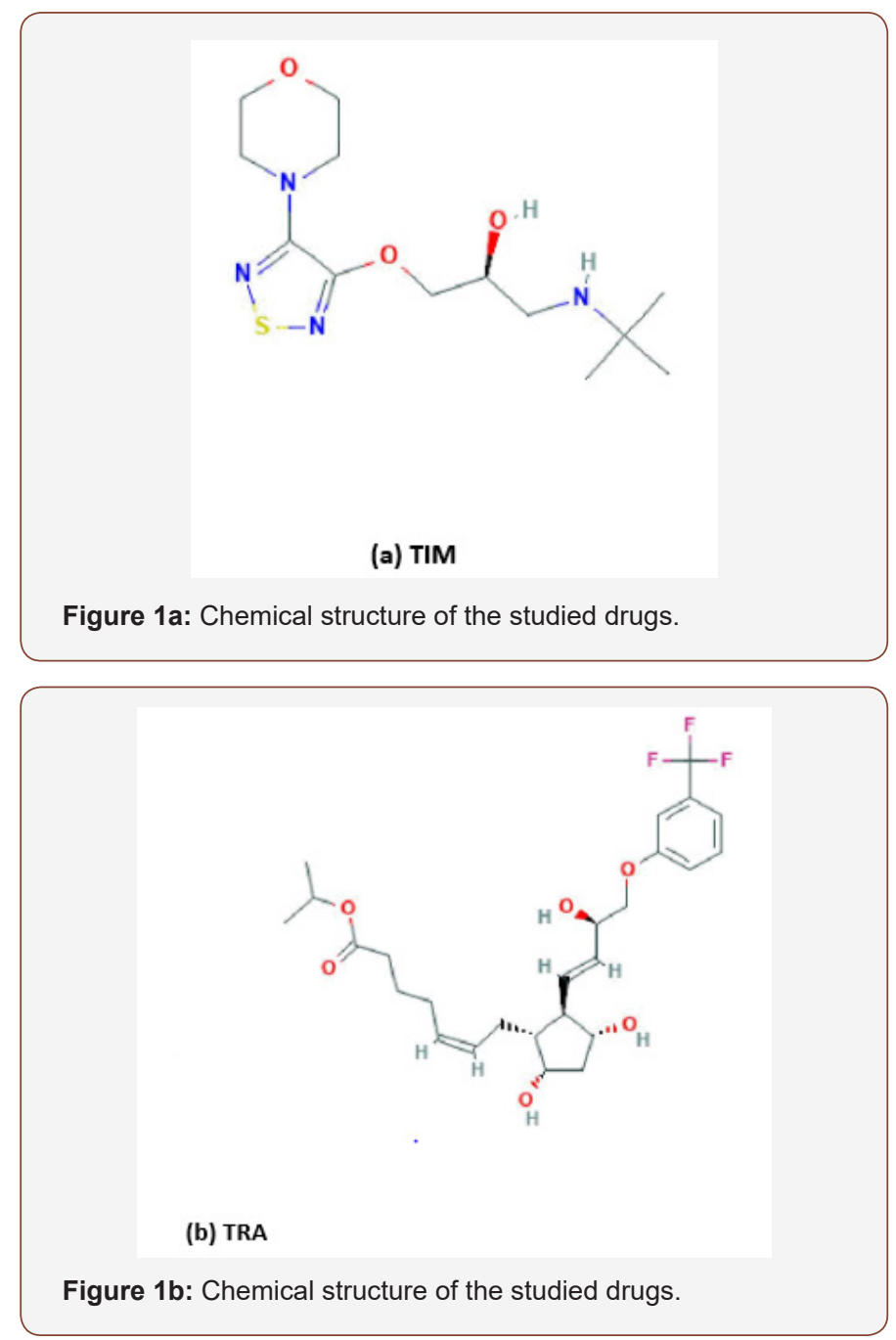

\section{Experimental}

\section{Apparatus}

Shimadzu-UV 1800 double beam UV-Visible spectrophotometer (Japan) with matched $1 \mathrm{~cm}$ quartz cells at 200-400 nm range was used for all absorbance measurements. Spectra were automatically obtained by Shimadzu UV-Probe 2.32 system software. The data points for the zeroorder spectra were then collected at every 0.1 $\mathrm{nm}$. All computations were performed in Matlab for WindowsTM version 6.5. The PLS procedure was taken from PLS-Toolbox 2.0 for use with Matlab ${ }^{\circledR}$ 7.9. TLC-spectrodensitometric system: CAMAG TLC scanner 3 S/ N 130319 operated with winCATS software, Linomat 5 autosampler (CAMAG, Muttenz, Switzerland), CAMAG micro syringe $(100 \mu \mathrm{L})$. TLC aluminum sheets $(20 \times 20 \mathrm{~cm})$ precoated with silica gel 60 F254 (Merck

KgaA, Darmstad, Germany) were used. Calculations were performed using the Microsoft Excel program. The centrifugation system: Laboratory Centrifuge, Sigma 2-16KL, Sigma 2-16KHL, with order number 10350, 10353.

\section{Materials and reagents}

Pharmaceuticals: The investigated drugs were obtained and were used as such without further treatments : TIM (E.I.P.I.CO., Cairo, Egypt), TRA (Novartus Co., Egypt) with general estimated purity $99.44 \% \mathrm{w} / \mathrm{w}$ and $99.18 \% \mathrm{w} / \mathrm{w}$ on dried basis for TIM and TRA [1], respectively.

Solvents and reagents: Methanol (S.D. Fine-Chem Limite, India) was of analytical grade. Ethyl acetate and ammonia 30\% (ElNasr Pharmaceutical Co., Abu Zaabal, Egypt). In addition, Whatman No. 1 filter paper $110 \mathrm{~mm}$ diameter (Sigma-Aldrich) was used for filtration of sample solutions.

Pharmaceutical formulations: The pharmaceutical preparation; Avatan-T® eye drops labeled to contain $40 \mu$ g and 5 mg TRA and TIM per mL., respectively, manufactured by (Alcon, Co., Belgium).

\section{Methodology}

Standard preparations: Standard stock solutions containing 0.5 mg.mL-1 of either TRA or TIM were prepared separately by dissolving the reference materials in methanol. Regarding the stability of solutions of drugs, stock solutions were stored at room temperature and were found to be stable for at least 10 days.

Working preparations: Preparation of working solutions was done by diluting the standard solutions with methanol to give concentrations in the range $0.4-50 \mu \mathrm{g} . \mathrm{mL}-1$ for either TRA or TIM.

Synthetic mixtures: Accurate volumes of standard solutions of TRA and TIM were transferred into two sets each of three $10-\mathrm{mL}$ volumetric flasks then completed to volume with methanol so that the final concentration of each drug was within its linearity range as cited in Table 1.

\section{Sample preparation}

- $\quad$ Eye drops sample: Dilute an appropriate volume of the solution in a volumetric flask so as to contain $40 \mu \mathrm{g}$ and $5 \mathrm{mg}$ TRA and TIM, respectively. Proceed as directed under 4.3.1. and 4.3.2.

\section{General procedure}

Spectro-chemometric methods: Different working standard solutions were prepared with known concentrations within the calibration range specified for each drug as shown in Table 1. These ranges were confirmed to obey Beer's law for each of the cited drugs in methanol. These concentrations were used to construct calibration matrix which can be used for the direct determination of the current drugs by the different used techniques; (First derivative spectrophotometric (1D) and isosbestic point methods. The obtained absorption data in the general range of 200-350 nm (digitalized every $1.0 \mathrm{~nm}$ ), against reagent blank treated similarly Figure 2, were stored in PC apparatus program. The spectral data were transferred to Microsoft excel 2003 program by performing 
statistical manipulation and processing them with the Standard curve fit package and matrix calculations and then were subjected to statistical treatment specified for each technique (Table 1) (Figure 2).

Table 1: Analytical parameters of the proposed methods

\begin{tabular}{|c|c|c|c|c|c|c|c|c|c|c|}
\hline Method & $\begin{array}{l}\text { Linearity range } \\
\left(\mu \mathrm{gmL}^{-1}\right)\end{array}$ & $\mathbf{a}$ & $S_{a}$ & b & $S_{b}$ & $\mathbf{r}$ & $\mathbf{r}^{2}$ & $\begin{array}{c}\text { LOD } \\
\left(\mu \mathrm{gmL}^{-1}\right)\end{array}$ & $\begin{array}{c}\text { LOQ } \\
\left(\mu \mathrm{gmL}^{-1}\right)\end{array}$ & RMSEP \\
\hline \multicolumn{11}{|l|}{${ }^{1} \mathbf{D}$} \\
\hline TIM (226 nm) & $0.4-50$ & 0.0579 & 0.0075 & 0.0593 & $1.99 \times 10^{-5}$ & 0.9998 & 0.9996 & 0.113 & 0.373 & \\
\hline TRA $(292 \mathrm{~nm})$ & $0.4-50$ & 0.0819 & 0.0053 & 0.0844 & $1.07 \times 10^{-5}$ & 0.9999 & 0.9998 & 0.0985 & 0.325 & \\
\hline \multicolumn{11}{|l|}{ Isosbestic } \\
\hline$(230.5 \mathrm{~nm})$ & $0.4-50$ & 0.4007 & 0.0028 & 0.7826 & $1.66 \times 10^{-5}$ & 0.9992 & 0.9984 & 0.104 & 0.343 & \\
\hline$(272 \mathrm{~nm})$ & $0.4-50$ & 0.1033 & 0.0019 & 0.1986 & $0.83 \times 10^{-5}$ & 0.9994 & 0.9988 & 0.008 & 0.026 & \\
\hline \multicolumn{11}{|l|}{ CLS } \\
\hline TIM & $0.4-50$ & 0.8061 & 0.0086 & 0.9932 & $8.45 \times 10^{-5}$ & 0.9989 & 0.9978 & 0.111 & 0.366 & 0.8765 \\
\hline TRA & $0.4-50$ & 0.8973 & 0.0046 & 0.984 & $4.23 \times 10^{-5}$ & 0.999 & 0.998 & 0.107 & 0.353 & 0.439 \\
\hline \multicolumn{11}{|l|}{ PLS } \\
\hline TIM & $0.4-50$ & 0.5044 & 0.007 & 1.0012 & $6.39 \times 10^{-5}$ & 0.9986 & 0.9972 & 0.018 & 0.059 & 0.703 \\
\hline TRA & $0.4-50$ & 0.0238 & 0.0013 & 1.0109 & $16.6 \times 10^{-5}$ & 0.9984 & 0.9968 & 0.106 & 0.349 & 0.4385 \\
\hline \multicolumn{11}{|l|}{ PCR } \\
\hline TIM & $0.4-50$ & 0.1345 & 0.0065 & 0.9994 & $6.54 \times 10^{-5}$ & 0.9985 & 0.997 & 0.081 & 0.267 & 0.702 \\
\hline TRA & $0.4-50$ & 0.6893 & 0.0078 & 1.0129 & $4.11 \times 10^{-5}$ & 0.9989 & 0.9978 & 0.101 & 0.333 & 0.4589 \\
\hline \multicolumn{11}{|l|}{ HPTLC } \\
\hline TIM & $0.4-50$ & -56.2 & 80.05 & 100.2 & 2.87 & 0.9993 & 0.9986 & 0.087 & 0.287 & \\
\hline TRA & $0.4-50$ & -194 & 27.67 & 290.29 & 1.76 & 0.9992 & 0.9984 & 0.1 & 0.333 & \\
\hline
\end{tabular}

a-Intercept, $S_{a}-S D$ of intercept, r-correlation coefficient, $r^{2}$-Determination coefficient.

b-Slope, $\mathrm{S}_{\mathrm{b}}$-SD of slope, LOD-Lower limit of detection, LOQ-Lower limit of quantitation

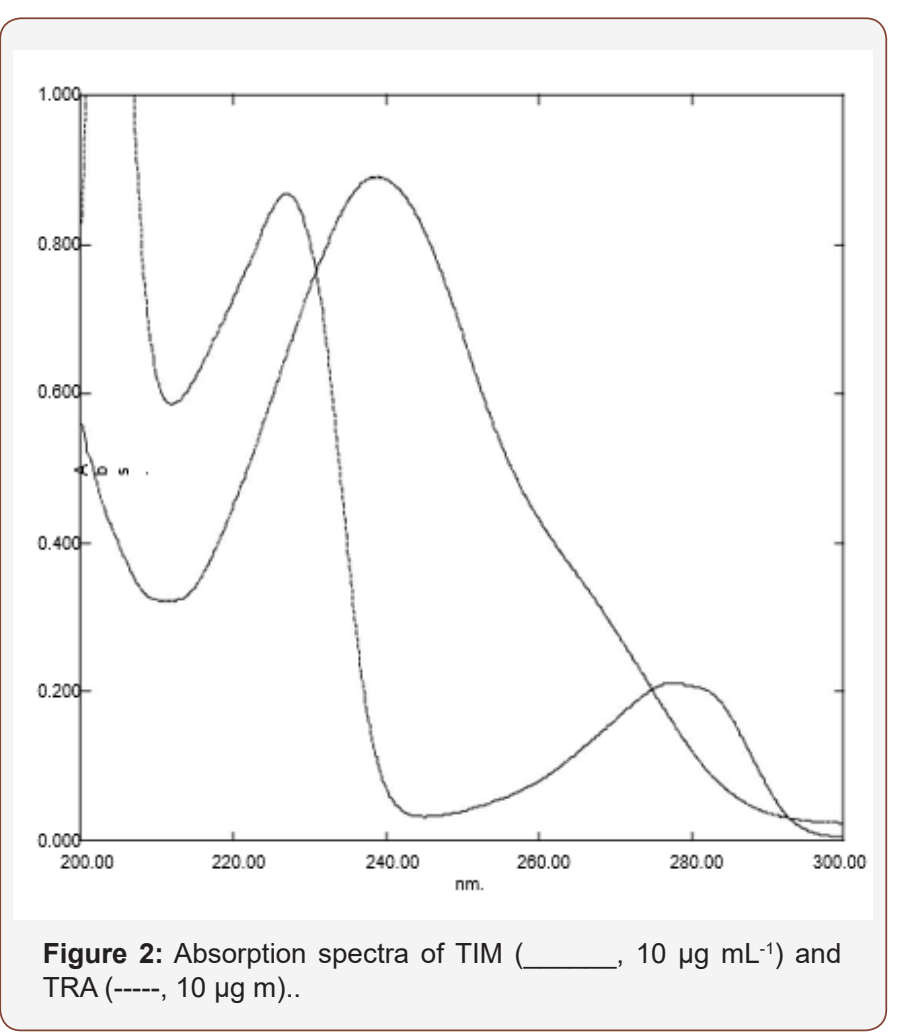

a) First derivative spectrophotometric (1D) method: 1D values of TIM were measured at the zero crossing point of TRA at $226 \mathrm{~nm}$. The 1D value of TRA was measured at $292 \mathrm{~nm}$. For each drug, the calibration graphs were plotted demonstrating the concentration values of $1 \mathrm{D}$ amplitude and the regression equations were computed which is further used for calculation of each drug in the sample preparation.

b) Isosbestic point method: The zero order absorption spectra of $10 \mu \mathrm{g} \mathrm{mL-10f}$ each of the studied drugs were recorded as shown in Figure 2. In order to relate the absorbance of the zero order spectra of TIM at their isosbestic points; 230.5 or $272 \mathrm{~nm}$ to its corresponding concentrations in $\mu \mathrm{g} \mathrm{mL}-1$. Calibration curves were plotted and the regression equation at each wavelength was derived, illustrated in Table 2. In order to analyze the studied drugs in their binary mixture, different volumes of TRA and TIM were precisely withdrawn from their corresponding working solutions and pipetted into $10 \mathrm{~mL}$ volumetric flask to make solutions containing different ratios of each two drugs in their binary mixtures as shown in Figure 2 and then the resultant solution was made up to volume with the same solvent. To calculate the concentration of the studied drugs in their binary mixture, the absorbance of the resulting solution at (1D) $226 \mathrm{~nm}$ represented the content of TIM only, which 
can be obtained using its derived equation at same wave length as shown in Table 2. The total content of each of the prepared binary mixture was determined at their isosbestic points; 230.5 or 272 $\mathrm{nm}$ by measuring their corresponding absorbencies at these points and then applied the derived regression equation at each points

Table 2: Evaluation of the accuracy and precision of the proposed methods

\begin{tabular}{|c|c|c|c|c|c|c|}
\hline \multirow{3}{*}{ Method } & \multicolumn{6}{|c|}{ Amount taken } \\
\hline & \multicolumn{2}{|c|}{$20 \mu \mathrm{g} \mathrm{mL}^{-1} \%$ Recovery \pm SD (RSD) } & \multicolumn{2}{|c|}{$35 \mu \mathrm{g} \mathrm{mL}^{-1} \%$ Recovery \pm SD (RSD) } & \multicolumn{2}{|c|}{$50 \mu \mathrm{g} \mathrm{mL}^{-1} \%$ Recovery \pm SD (RSD) } \\
\hline & Intraday & Interday & Intraday & Interday & Intraday & Interday \\
\hline \multicolumn{7}{|l|}{${ }^{1} \mathbf{D}$} \\
\hline $\begin{array}{l}\text { TIM (226 } \\
\text { nm) }\end{array}$ & $99.67 \pm 0.37(0.37)$ & $99.28 \pm 0.46(0.46)$ & $100.45 \pm 0.68(0.68)$ & $100.18 \pm 0.77(0.77)$ & $99.09 \pm 0.87(0.87)$ & $99.168 \pm 0.82(0.82)$ \\
\hline $\begin{array}{c}\text { TRA (292 } \\
\text { nm) }\end{array}$ & $99.28 \pm 0.97(0.97)$ & $99.93 \pm 0.71(0.71)$ & $99.49 \pm 0.55(0.55)$ & $99.99 \pm 0.79(0.79)$ & $99.49 \pm 0.77(0.77)$ & $99.93 \pm 0.71(0.71)$ \\
\hline \multicolumn{7}{|l|}{ Isosbestic } \\
\hline $\begin{array}{c}(230.5 \\
\mathrm{nm})\end{array}$ & $99.94 \pm 0.49(0.49)$ & $99.32 \pm 0.74(0.74)$ & $99.67 \pm 0.83(0.83)$ & $100.18 \pm 0.77(0.77)$ & $100.47 \pm 0.28(0.28)$ & $100.54 \pm 0.19(0.19)$ \\
\hline$(272 \mathrm{~nm})$ & $99.76 \pm 0.88(0.88)$ & $100.08 \pm 0.75(0.75)$ & $99.22 \pm 0.65(0.65)$ & $99.76 \pm 0.49(0.49)$ & $99.44 \pm 0.25(0.25)$ & $99.47 \pm 0.91(0.91)$ \\
\hline \multicolumn{7}{|l|}{ CLS } \\
\hline TIM & $99.55 \pm 0.78(0.78)$ & $100.12 \pm 0.49(0.49)$ & $99.45 \pm 0.48(0.48)$ & $99.27 \pm 0.29(0.29)$ & $99.56 \pm 0.54(0.54)$ & $99.99 \pm 0.78(0.78)$ \\
\hline TRA & $99.39 \pm 0.84(0.84)$ & $99.96 \pm 0.55(0.55)$ & $99.49 \pm 0.39(0.39)$ & $99.33 \pm 0.71(0.71)$ & $99.35 \pm 0.84(0.84)$ & $99.56 \pm 0.83(0.83)$ \\
\hline \multicolumn{7}{|l|}{ PLS } \\
\hline TIM & $99.24 \pm 0.98(0.98)$ & $99.09 \pm 0.32(0.32)$ & $99.23 \pm 0.66(0.66)$ & $99.87 \pm 0.97(0.97)$ & $99.55 \pm 0.89(0.89)$ & $99.13 \pm 0.59(0.59)$ \\
\hline TRA & $99.56 \pm 0.76(0.76)$ & $99.32 \pm 0.65(0.65)$ & $99.59 \pm 0.93(0.93)$ & $99.21 \pm 0.45(0.45)$ & $99.22 \pm 0.76(0.76)$ & $99.56 \pm 0.39(0.39)$ \\
\hline \multicolumn{7}{|l|}{ PCR } \\
\hline TIM & $99.87 \pm 0.56(0.56)$ & $99.2 \pm 0.59(0.59)$ & $99.56 \pm 0.91(0.91)$ & $100.54 \pm 0.86(0.86)$ & $99.67 \pm 0.49(0.49)$ & $100 \pm 0.29(0.29)$ \\
\hline TRA & $99.3 \pm 0.77(0.77)$ & $99.44 \pm 0.83(0.83)$ & $99.29 \pm 0.78(0.78)$ & $99.65 \pm 0.89(0.89)$ & $99.87 \pm 0.87(0.87$ & $99.09 \pm 0.49(0.49)$ \\
\hline \multicolumn{7}{|l|}{ HPTLC } \\
\hline TIM & $99.55 \pm 0.97(0.97)$ & $99.48 \pm 0.81(0.81)$ & $99.55 \pm 0.76(0.76)$ & $99.55 \pm 0.84(0.84)$ & $99.18 \pm 0.43(0.43)$ & $99.88 \pm 0.86(0.86)$ \\
\hline TRA & $100.08 \pm 0.39(0.39)$ & $99.99 \pm 0.94(0.94)$ & $99.28 \pm 0.77(0.77)$ & $99.65 \pm 0.84(0.84)$ & $99.97 \pm 0.19(0.7619)$ & $99.16 \pm 0.54(0.54)$ \\
\hline
\end{tabular}

SD: Standard deviation, RSD: Relative standard deviation

Multivariate chemometric methods: Prepare twenty five mixtures of TIM and TRA standard solution; seventeen mixtures were used for building the calibration model, while eight mixtures were chosen to be used as an external validation set. The laboratory prepared mixtures of each of the TIM and TRA were prepared in a concentration range of (0.4-50 $\mu \mathrm{g} \mathrm{mL}-1)$ to reach the concentrations listed in Table 3. The absorbance of these mixtures were measured between 200 and $400 \mathrm{~nm}$ at $1 \mathrm{~nm}$ intervals with respect to a blank of methanol. Several multivariate calibration models were provided in Table 2. TRA concentration was calculated at each points by subtraction of the concentration of TIMA, determined at (1D) $226 \mathrm{~nm}$, from the total concentrations of both drugs at their isosbestic points (Table 2).

Table 3: Concentrations of TIM and TRA in the calibration and validation set using PCR, PLS and CLS methods

\begin{tabular}{|c|c|c|}
\hline Mixture No. & TIM $\left(\mu \mathrm{g} \mathrm{mL} \mathrm{L}^{-1}\right)$ & TRA $\left(\mu \mathrm{g} \mathrm{mL}^{-1}\right)$ \\
\hline $1^{\mathrm{a}}$ & 50 & 0.4 \\
\hline 2 & 20 & 50 \\
\hline 3 & 40 & 10 \\
\hline 4 & 50 & 30 \\
\hline 5 & 30 & 50 \\
\hline 6 & 40 & 0.4 \\
\hline 7 & 50 & 10 \\
\hline 8 & 20 & 40 \\
\hline 9:00 AM & 30 & 40 \\
\hline
\end{tabular}




\begin{tabular}{|c|c|c|}
\hline $10: 00 \mathrm{AM}$ & 10 & 30 \\
\hline 11 & 50 & 20 \\
\hline 12 & 0.4 & 20 \\
\hline $13^{\mathrm{a}}$ & 30 & 30 \\
\hline 14 & 40 & 40 \\
\hline 15 & 20 & 50 \\
\hline 16 & 50 & 30 \\
\hline $17^{\mathrm{a}}$ & 10 & 10 \\
\hline 18 & 40 & 10 \\
\hline 19 & 20 & 50 \\
\hline 20 & 30 & 20 \\
\hline $21^{\mathrm{a}}$ & 40 & 0.4 \\
\hline 22 & 0.4 & 30 \\
\hline 23 & 20 & 0.4 \\
\hline $24^{\mathrm{a}}$ & 30 & 0.4 \\
\hline
\end{tabular}

\section{A Mixtures of validation set}

(TLC)-Densitometric methods: The TLC densitometric method was used for the determination of TIM and TRA by separation from each other, depending on the difference in $\mathrm{Rf}$ values. Complete separation of TIM from TRA was achieved using ethylacetate:methanol:ammonia (5:3.0:0.5, v/v/v) as the mobile phase. Densitometric scanning was performed at $274 \mathrm{~nm}$ with accepted results (Figure $3 \& 4$ ).

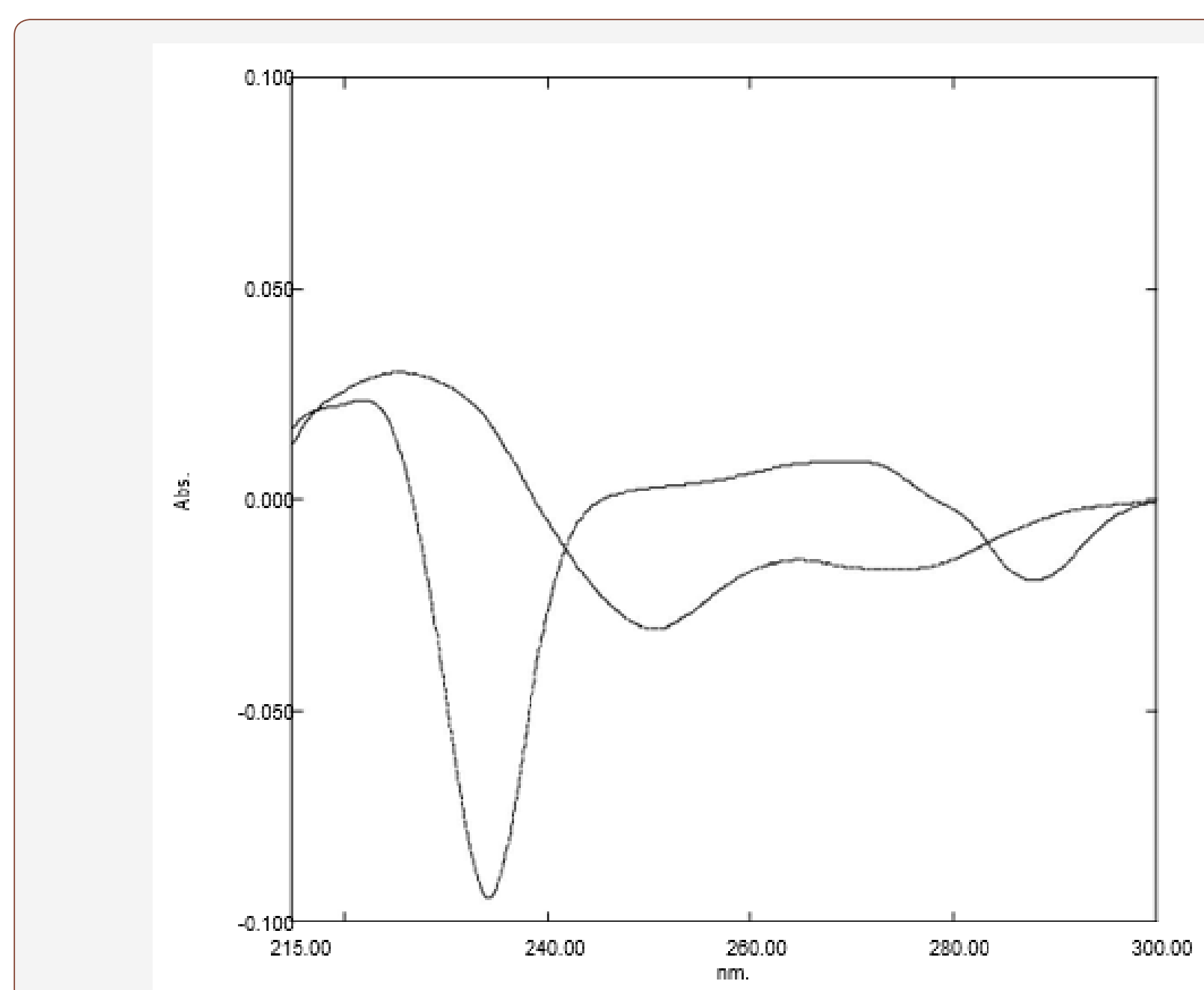

Figure 3: ${ }^{1} \mathrm{D}$ spectra of TIM ( , $\left.10 \mu \mathrm{g} \mathrm{mL}^{-1}\right)$ and TRA (-----, $\left.10 \mu \mathrm{g} \mathrm{mL}-1\right)$. 


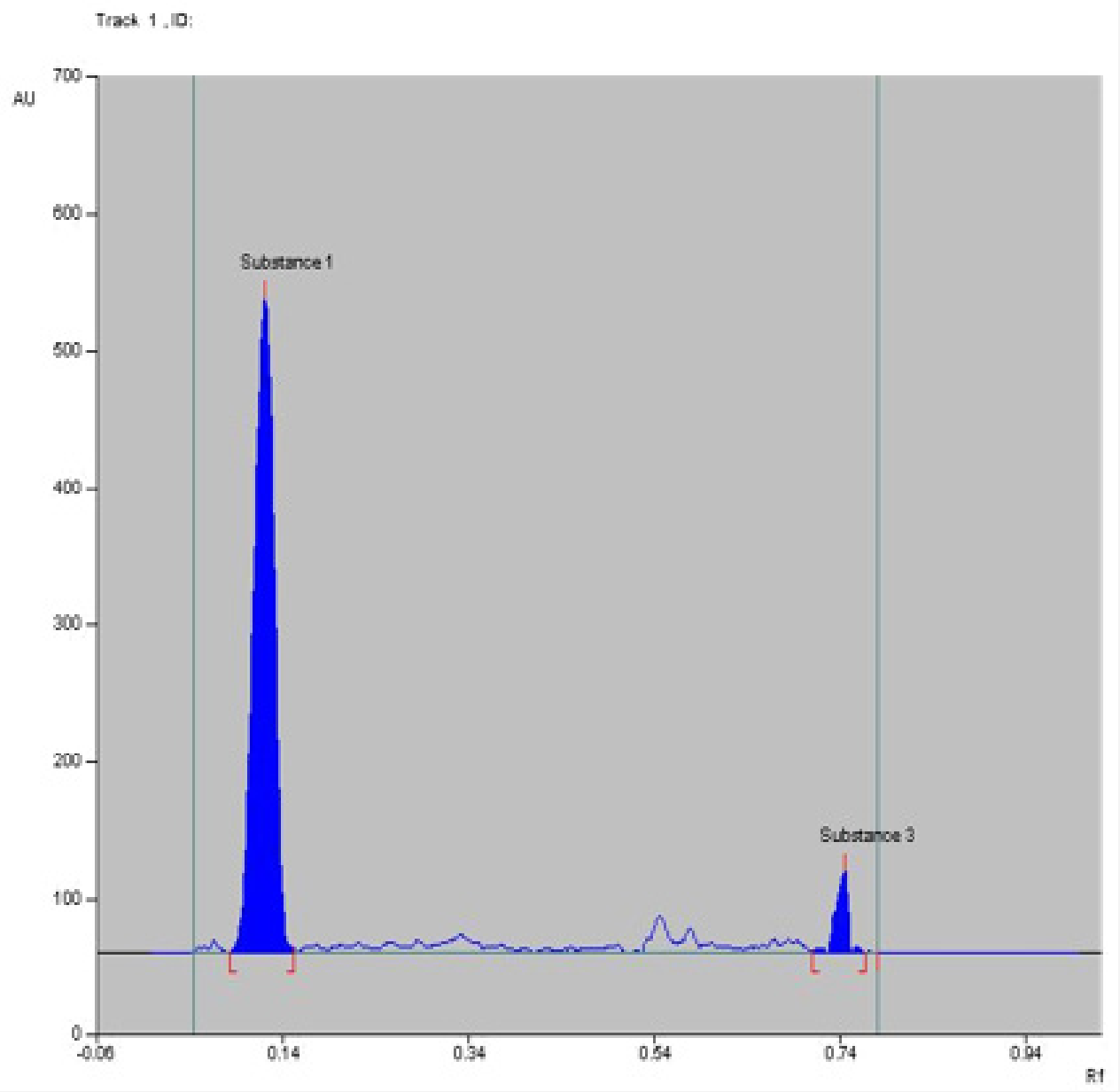

Figure 4: HPTLC chromatographic separation of TIM $\left(50 \mu \mathrm{g} \mathrm{mL}^{-1}\right)$ and TRA $\left(0.4 \mu \mathrm{gL}^{-1}\right)$.

\section{Results and Discussions}

\section{Optimization of variables}

\section{Spectro-chemometric method}

Spectro-chemometric methods were considered the methods of choice for assay of two component mixtures [24-29]. In this work two spectro-chemometric method such as first derivative (1D) and isosbestic point procedures were developed for the direct determination of TIM and TRA in their dosage form preparation.

Selection of solvent used: Methanol was the solvent of choice for this mixture, where it was found to be the most suitable one for both TIM and TRA from their dosage form. In addition, significant decrease in the degree of overlapping between the two mixture components and higher sensitivity were also observed.

Stability study: The absorbance of the studied drugs was found to be stable for $60 \mathrm{~min}$ within $200-350 \mathrm{~nm}$ range.

First derivative spectrophotometric (1D) method: In this work, first derivative spectrophotometry was examined for the assay of two component drug mixture. Figure 2 showed that TIM absorption spectrum is overlapped with that of TRA, which in turn made them difficult to be determined simultaneously. In the corresponding 1D curve, provided in Figure 3, the TIM 1D values were located at the zero crossing point of TRA at $226 \mathrm{~nm}$. TRA exhibited a 1D value at $292 \mathrm{~nm}$ where TIM first derivative value was nil (Figure 3).

Isosbestic point method: This method is based on the fact that the total concentration of the mixture was determined at their isosbestic points; 230.5 or $272 \mathrm{~nm}$. As illustrated in Figure 2 , Thus TIM concentration was determined by the application of first derivative spectrophotometric technique by measuring the absolute value of absorption spectra at its 1D, Kmax $226 \mathrm{~nm}$ without any interference from TRA. Then the concentration of TRA in the accompanied mixture at 230.5 or $272 \mathrm{~nm}$ was calculated as explained under 4.4.1.2.

\section{Multivariate Chemometric methods}

Among the different regression method existed for multivariate calibration, the factor analysis based methods including partial least squares (PLS regression and principal component regression (PCR) have received considerable attention in the chemometrics literature [30-34]. Due to the overlapped spectra of the drugs, (Figure 2), the previous chemometric methods have been used to analyze this mixture. An appropriate choice of the number of principal components or factors is necessary for PCR, PLS and CLS calibrations. The number of factors should account as much as possible for the experimental data without resulting in over fitting. Various criteria have been developed to select the optimum number [35]. Cross-validation methods leaving out one sample at a time was employed [36]. The predicted concentrations were compared with 
the known concentrations of the compounds in each calibration sample. The root mean squares error of cross-validation (RMSECV) was calculated for each method for examining the errors in the predicted concentrations. The optimum number of factors was selected by following the criterion of Haal and and Thomas [37]. The selected model was that with the smallest number of factors such that RMSECV for that model was not significantly greater than RMSECV from the model with additional factor. A number of factors were found to be optimum for the mixture of TIM and TRA using PLS, PCR and CLS, Figure 5,6 respectively. To assess the prediction ability of the suggested models, an external validation set was used. The root mean squared error of prediction (RMSEP) values were calculated [38] Table 1. The percentage recoveries of the validation samples are shown in Table 2. The suggested methods were valid and applicable for the analysis of TIM and TRA in Avatan-T® eye drops Table 4. The validity of the proposed method is further assessed by applying the standard addition technique. The results obtained are shown in Table 5 (Table $4 \& 5$ and Figure 5\&6).

Table 4: Determination of the studied drugs in their pharmaceutical dosage form using the proposed methods compared to reference method.

\begin{tabular}{|c|c|c|c|c|c|}
\hline \multicolumn{2}{|c|}{ Drug } & \multicolumn{3}{|c|}{ Proposed methods } & \multirow{2}{*}{$\begin{array}{c}\text { Reference method } \\
\% \text { recovery } \pm \mathrm{SD}\end{array}$} \\
\hline \multicolumn{2}{|c|}{ Avatan-T® eye drops B.N.66543 } & $\%$ recovery $\pm \mathrm{SD}$ & $t^{*}$ & $\mathrm{~F}^{*}$ & \\
\hline \multirow[t]{2}{*}{${ }^{1} \mathrm{D}$} & $\operatorname{TIM}(226 \mathrm{~nm})$ & $99.76 \pm 1.02$ & 1.15 & 1.44 & $98.09 \pm 0.85$ \\
\hline & TRA (292nm) & $98.32 \pm 0.95$ & 0.97 & 1.1 & $99.40 \pm 1.00$ \\
\hline \multirow[t]{2}{*}{ Isosbestic } & $(230.5 \mathrm{~nm})$ & $99.04 \pm 0.89$ & 0.87 & 1.09 & $98.09 \pm 0.85$ \\
\hline & $(272 \mathrm{~nm})$ & $99.00 \pm 0.98$ & 0.38 & 1.04 & $99.40 \pm 1.00$ \\
\hline \multirow[t]{2}{*}{ CLS } & TIM & $98.89 \pm 0.85$ & 0.8 & 1 & $98.09 \pm 0.85$ \\
\hline & TRA & $99.76 \pm 0.87$ & 0.27 & 1.32 & $99.40 \pm 1.00$ \\
\hline \multirow[t]{2}{*}{ PLS } & TIM & $98.87 \pm 0.68$ & 1.56 & 1.56 & $98.09 \pm 0.85$ \\
\hline & TRA & $99.01 \pm 0.57$ & 0.12 & 3.07 & $99.40 \pm 1.00$ \\
\hline \multirow[t]{2}{*}{ PCR } & TIM & $99.78 \pm 0.45$ & 0.47 & 3.56 & $98.09 \pm 0.85$ \\
\hline & TRA & $99.57 \pm 0.76$ & 0.09 & 1.73 & $99.40 \pm 1.00$ \\
\hline \multirow[t]{2}{*}{ HPTLC } & TIM & $99.68 \pm 1.08$ & 0.98 & 1.61 & $98.09 \pm 0.85$ \\
\hline & TRA & $98.89 \pm .87$ & 0.38 & 1.32 & $99.40 \pm 1.00$ \\
\hline
\end{tabular}

*Tabulated value for the proposed methods and reported HPLC method $(n=4)$ at $95 \%$ confidence limit; $t=2.45$ and $F=9.28$.

Table 5: Determination of the studied drugs in their pharmaceutical dosage form using standard addition technique.

\begin{tabular}{|c|c|c|c|c|}
\hline Avatan-T ${ }^{\circledR}$ eye drops & Added $\mu \mathrm{g} \mathrm{mL}^{-1}$ & Found $\mu \mathrm{g} \mathrm{mL}^{-1}$ & Recovery $\%$ of added & Mean \pm SD \\
\hline \multirow[t]{3}{*}{${ }^{1} \mathbf{D}$} & 10 & 9.92 & 99.2 & $99.36 \pm 0.36$ \\
\hline & 20 & 19.86 & 99.32 & \\
\hline & 30 & 29.87 & 99.56 & \\
\hline \multirow[t]{3}{*}{ Isosbestic } & 10 & 9.92 & 99.21 & $99.49 \pm 0.49$ \\
\hline & 20 & 19.93 & 99.65 & \\
\hline & 30 & 29.89 & 99.63 & \\
\hline \multirow[t]{3}{*}{ CLS } & 10 & 9.98 & 99.8 & $99.30 \pm 0.34$ \\
\hline & 20 & 19.8 & 99 & \\
\hline & 30 & 29.77 & 99.23 & \\
\hline \multirow[t]{3}{*}{ PLS } & 10 & 10.08 & 100.8 & $100.76 \pm 0.76$ \\
\hline & 20 & 20.19 & 100.95 & \\
\hline & 30 & 30.16 & 100.53 & \\
\hline \multirow[t]{3}{*}{ PCR } & 10 & 9.86 & 98.65 & $99.06 \pm 0.73$ \\
\hline & 20 & 19.79 & 98.95 & \\
\hline & 30 & 29.88 & 99.6 & \\
\hline \multirow[t]{3}{*}{ HPTLC } & 10 & 9.99 & 99.9 & $99.38 \pm 0.72$ \\
\hline & 20 & 19.79 & 98.95 & \\
\hline & 30 & 29.79 & 99.31 & \\
\hline
\end{tabular}




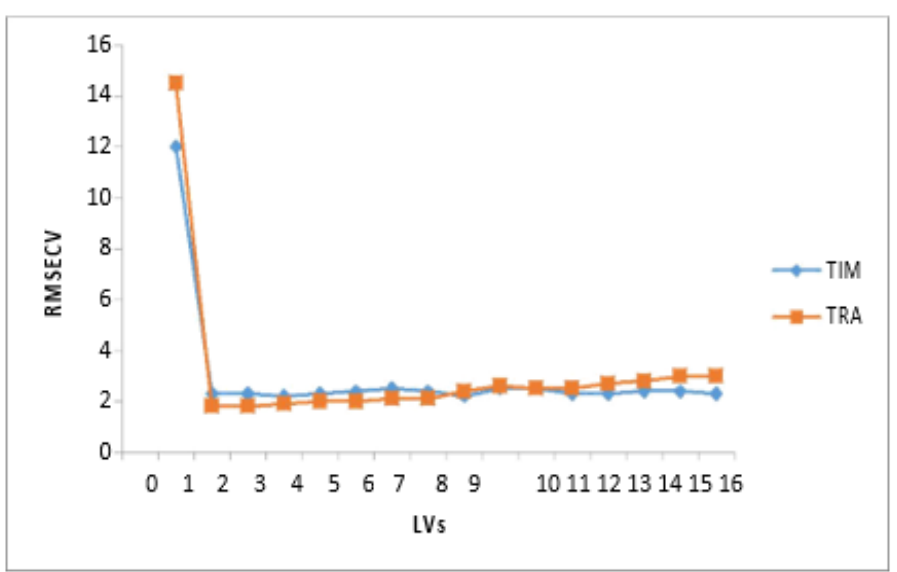

Figure 5: RMSECV plot of the crossvalidation results of the calibration set as a function of the number of latent variables (LVs) used to construct the PCR model.

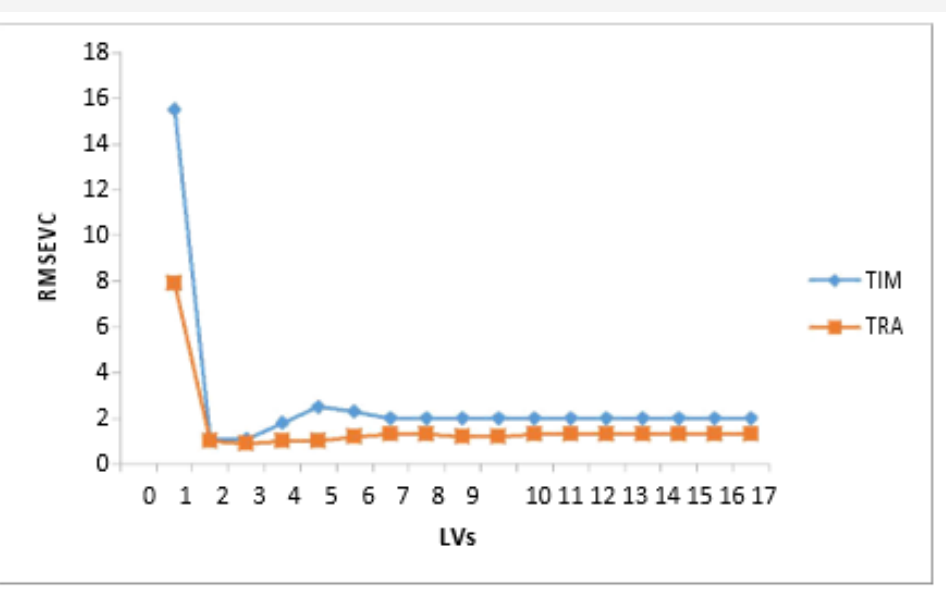

Figure 6: RMSECV plot of the crossvalidation results of the calibration set as a function of the number of latent variables (LVs) used to construct the PLS model.

Table 6: Statistical analysis of parameters required for system suitability testing of TLC method.

\begin{tabular}{|c|c|c|c|}
\hline Parameter & TIM & TRA & Limit \\
\hline Retention factor $\left(\mathrm{R}_{\mathrm{f}}\right)$ & $0.13 \pm 0.02$ & $0.74 \pm 0.02$ & \\
\hline Resolution $\left(\mathrm{R}_{\mathrm{s}}\right)$ & & 6.55 & Rs $>2$ \\
\hline Tailing factor $(\mathrm{T})$ & 0.8 & 0.9 & $\mathrm{~T}<2$ \\
\hline Capacity factor $(\mathrm{K})$ & 54 & 50 & $\mathrm{~K}>2$ \\
\hline Selectivity factor $(\alpha)$ & & 18 & \\
\hline
\end{tabular}

\section{(TLC)-Densitometric methods}

The current work discussed the implementation of a newly developed HPTLC with regard to direct determination of TIM and TRA in their dosage form preparation without prior separation.

Optimization: The TLC densitometric method was used for the determination of TIM and TRA by separation from each other, depending on the difference in Rf values. Complete separation products was achieved using ethylacetate:methanol:ammonia (5.0:3.0:0.5, v/v/v) as the mobile phase. Densitometric scanning was performed at $274 \mathrm{~nm}$ with accepted results Figure 4.

Solution stability: The stability of TRA and TIM in their both standard and pharmaceutical preparation solutions was investigated. This was done by storing solutions of the two drugs at room temperature for different time intervals (1, 3 and $5 \mathrm{~h}$ ) before being analyzed by the proposed method. No decomposition of the drugs was observed during development which was confirmed by the absence of any additional peaks in the chromatograms obtained throughout the analysis time together with the nearly unchangeable peak area of the drugs during stability studies (RSD \% less than $2 \%$ ) indicating stability of the drugs solutions for at least $5 \mathrm{~h}$, which was enough for completing the analytical procedure.

Spot stability: The time for which the sample stands on the plate between spotting and chromatographic development can affect its stability and should be assessed for validation. Twodimensional chromatography was applied to study decomposition occurring during spotting and development. Appearance of peak (s) of decomposition product (s) in both the first and second development directions indicates decomposition during development. Using the proposed conditions, no decomposition was observed during spotting and development indicating stability of the four drugs in their solutions.

\section{Validation of the current procedures}

The suggested spectro-chemometric, multivariate chemometric and HPTLC procedures were validated according to USP [39] and ICH [40] guidelines. 
Linearity and analytical parameters: Upon application of the optimized experimental conditions, for all the procedures studied, the least-squares regression analysis was applied to compute the regression equations for each drug under study as explained under the general procedure 2.4., and the results obtained are summarized in Table 1. In all cases, the analytical parameters such as linear range, the intercepts (a), slopes (b), correlation coefficients (r), determination coefficients (r2), limits of detection (LOD) and limits of quantitation (LOQ) are included. The slopes are used as measure of sensitivity of the suggested methods, while intercepts indicate the magnitude of background interferences. In all methods applied, the results listed in Table 1 indicate the high sensitivity and low background effects. The obtained values of correlation coefficients ( $r$ ) and determination coefficients (r2) confirmed a linear relationship between the response measured at the chosen wavelength(s) and the selected concentrations of both drugs in the range specified.

Accuracy and precision: Accuracy and precision of the current procedures were studied at three various levels of the cited drugs concentrations. In this study each of the chosen concentration was analyzed six times by the proposed methods. Table 2 summarizes the results of such study. These results were quite satisfactory reflecting good accuracy of the current procedures. The computed relative standard deviations were found to be below $2 \%$ for all procedures studied proving perfect precision of all suggested methods. In addition, the suggested methods were used for the analyses of TIM and TRA in prepared laboratory mixtures with various ratios of both drugs ranged from 1: 1 to 1:125. The percentage recoveries in all cases were satisfactory and the calculated relative standard deviation values for both drugs were below 2 reflecting good accuracy and precision of the suggested procedures.

Specificity and interference: This study has been done by all the proposed methods through analysis of pharmaceutical preparation extract by standard addition method where the samples under study were spiked separately with certain concentration of the investigated drugs in different ratios. In all cases, satisfactory $\%$ recovery with relative standard deviation value for both drugs did not exceed 2 were obtained; indicating that no interference is observed from the matrix background.

Analyses of available dosage form: The suggested procedures were used for the direct estimation of TIM and TRA in Avatan-T® eye drops and the results obtained were contrasted with those obtained upon application of reference method [1] as illustrated in Table 1. Statistical analysis of the obtained results using Fand $t$-tests shows that there is no remarkable differences at $95 \%$ confidence level. As can be seen, the percentage recoveries in all cases were satisfactory for both drugs reflecting good accuracy, precision and the applicability of the suggested methods to the pharmaceutical preparations.

Robustness: Robustness of the proposed methods was investigated by studying the effect of small change in the experimental parameter(s) on the analytical performance of the method. In this study the parameter under study was changed while the others were kept the same. In case of chemometric and HPTLC methods, variation of wavelength of detection $\pm 2 \mathrm{~nm}$ were tried. In all cases the general procedure was applied and the percentage recovery was computed. It was clear that small variation of the variables studied has no significant influence the efficiency of the method under study. Therefore the proposed methods are reliable with regard to the analyses of the chosen drugs in either bulk forms or their dosage forms.

\section{Conclusion}

The current research work describes simple, sensitive, reliable, accurate, reproducible, time saving and economic two spectro-Chemometric, three multivariate chemometric and HPTLC procedures with regard to the direct estimation of the studied drugs in their binary mixture and in the available dosage form. The most important features of these methods are no tedious sample preparation or treatment such as heating, derivatization which may needed especially for HPLC procedure. For HPTLC the cited drugs were detected simultaneously within a period of time less than 8 min. The current procedures were evaluated according to USP and ICH rules where acceptable regression values, RSD \% and standard deviations were observed, thus make them versatile and valuable for simultaneous estimation of two drugs in their formulation. Data obtained after analysis of the studied formulations by the suggested methods were too close to label claims, which reflect absence of interference from the accompanied excipients during estimation of the cited drugs by the proposed procedures. Owing to the suitability of the suggested procedures with regard to the routine analyses of the dosage forms of these drugs, they may be used in quality control for these products.

\section{Acknowledgement}

None.

\section{Conflict of Interest}

No conflict of interest.

\section{References}

1. Jothieswari D, Reddy BCO, Soniya S, Dharshini Y, Roja K (2017) Method development and validation of simultaneous estimation of Timolol maleate and Travoprost in bulk and in pharmaceutical dosage form by UV-spectroscopy. J Pharm. Bio 7(1)L: 22-32.

2. Walash MI, Toubar S, Abou El-Alamin MM, Elabd MA, Salama NN (2018) Green spectrophotometry and spectrofluorimetric methods for rapid economic determination of travoprost: study on inclusion complex with beta cyclodextrin. Iajps 05(03): 1327-1336.

3. Lotfy HM, Hegazy MA, Rezk MR, Omran YR (2014) Novel spectrophotometric methods for simultaneous determination of timolol and dorzolamide in their binary mixture. Spectrochim Acta A Mol Biomol Spectrosc 126: 197-207.

4. Desai HH, Captain AD (2014) Three simple validated UV spectrophotometric methods for the simultaneous estimation of timolol maleate and brimonidine tartrate and their comparison using anova. Int J Pharm Chem Bio Sci 4(3): 168-177.

5. Annapurna MM, Sushmitha M, Sevyatha VS (2017) Simultaneous Determination of Brimonidine Tartrate and Timolol Maleate by First 
Derivative and Ratio Derivative Spectroscopy. J Anal Pharm Res 4(6): 120.

6. Nnadi CO, Obonga WO, Ogbonna JDN, Ugwa LO (2015) Development of Vanadometric System for Spectrophotometric Determination of Timolol in Pure and Dosage Forms. Trop J Pharm Res 14(12): 2223-2229.

7. Bebawy LI (2002) Application of TLC-densitometry, first-derivative UV-spectrophotometry and ratio derivative spectrophotometry for the determination of dorzolamide hydrochloride and timolol maleate. J Pharm Biomed Anal 27(5): 737-746.

8. Erk N (2002) Simultaneous determination of dorzolamide HCL and timolol maleate in eye drops by two different spectroscopic methods. J Pharm Biomed Anal 28(2): 391-397.

9. McCue BA, Cason MM, Curtis MA, Faulkner RD, Dahlin DC (2002) Determination of travoprost and travoprost free acid in human plasma by electrospray HPLC/MS/MS. J Pharm Biomed Anal 28(2): 199-208.

10. Marchei E, Orsi D, Guarino C, Rotolo MC, Graziano S, Pichini S (2016) High Performance Liquid Chromatography Tandem Mass Spectrometry Measurement of Bimatoprost, Latanoprost and Travoprost in Eyelash Enhancing Cosmetic Serums. Cosmetics 3(4): 1-8.

11. Anusha B, Susmita AG, Rajitha G (2016) Analytical Method Development and Validation of New RP-HPLC Method for Simultaneous Estimation of Brinzolamide and Timolol Maleate in Ophthalmic Solutions. Res J Pharm Boil Chem Sci 7(3): 1290-1298.

12. Depani JA, Chaudhary AB, Shweta M, Bhadani SM, Patel BD (2018) Development and validation of RP-HPLC method for simultaneous estimation of bimatoprost and timolol maleate. World J Pharm Pharm Sci 7(5): 741-750.

13. Annapurna MM, Narendra A, Deepika D (2012) Development and validation of RP-HPLC method for simultaneous determination of dorzolamide and timolol maleate in pharmaceutical dosage forms. J Chrom Separat Techniq 2(2): 81-87.

14. Khatun R, Islam SMA (2014) Development and validation of analytical method for simultaneous estimation of Brinzolamide and Timolol by HPLC from ophthalmic preparation. Int J Pharm Sci Res 5(3): 1001-1007.

15. Chengalva P, Parameswari SA, Reddy PJ (2016) Simultaneous quantification of travoprost and timolol maleate in pharmaceutical formulation by RP-HPLC. Int J Pharm Sci Res 7(4): 1724-1728.

16. Elkady EF, Fouad MA, Elsabour SA, Elshazly HM (2018) Synchronized Separation of Timolol from Some Prostaglandin Analogs (Bimatoprost, Latanoprost and Travoprost) for Determination in their Combined Pharmaceutical Formulations Using RP-HPLC. Anal Chem Let 8(1): 7687.

17. Kumari RV, Venkateswar Rao P, Sunitha A, Keerthy K (2015) Stability indicating RP-HPLC method for simultaneous estimation of travopost and timolol in bulk and pharmaceutical dosage forms. Int J Pharm Sci Res 5(3): 176-182.

18. Ankit A, Sunil T, Kashyap N (2013) Method development and its validation for quantitative simultaneous determination of Travoprost, Timolol and Benzalkonium Chloride in ophthalmic solution by RP-HPLC. JDDT 3(2): 26-30.

19. Kulkarni SP, Amin PD (2000) Stability indicating HPTLC determination of timolol maleate as bulk drug and in pharmaceutical preparations. J Pharm Biomed Anal 23(6): 983-987.

20. Eissa MS, Nour IM, Elghobashy MR, Shehata MA (2017) Validated TLCDensitometry Method for Simultanouse Determination of Brinzolamide and Timolol in Their Ophthalmic Preparation. Anal Chem Let 7(6): 805812.

21. Jain PS, Khatal RN, Jivani HN, Surana SJ (2011) Development and Validation of TLC-densitometry Method for Simultaneous Estimation of Brimonidine tartrate and Timolol maleate in Bulk and Pharmaceutical Dosage Form. J Chrom Sep Tech 2(3):1-5.

22. Abou Al Alamein AM, Hendawy HAM, Elabd NO (2019) Chemometricsassisted voltammetric determination of timolol maleate and brimonidine tartrate utilizing a carbon paste electrode modified with iron (iii) oxide nanoparticles. Microchem J 145: 313-329.
23. Hendawy HAM, Elwy HM, Fekry AM (2017) Electrochemical and chemometric determination of dorzolamide and timolol in eye drops using modified multiwall carbon nanotube electrode. Int J Pharm Pharm Sci 9(9): 43-50.

24. Omar MA, Badr El-Din KM, Salem H, Abdelmageed OH (2018) Novel kinetic spectrophotometric method for estimation of certain biologically active phenolic sympathomimetic drugs in their bulk powders and different pharmaceutical formulations. Spectrochim Acta A Mol Biomol Spectrosc 193: 310-317.

25. Lofty HM, Salem H, Abdelkawy M, Samir A (2015) Spectrophotometric methods for simultaneous determination of betamethasone valerate and fusidic acid in their binary mixture. Spectrochim Acta A Mol Biomol Spectrosc 140: 294-304.

26. Salem H, Mohamed DA (2015) comparative study of smart spectrophotometric methods for simultaneous determination of a skeletal muscle relaxant and an analgesic in combined dosage form. Spectrochim Acta A Mol Biomol Spectrosc 140: 166-173.

27. Mostafa AA, Salem H, Hegazy M, Ali O (2015) Evaluating the efficiency of spectral resolution of univariate methods manipulating ratio spectra and comparing to multivariate methods: An application to ternary mixture in common cold preparation. Spectrochim Acta A Mol Biomol Spectrosc 137: 1363-1373.

28. Lotfy HM, Saleh SS, Hassan NY, Salem H (2015) Novel two wavelength spectrophotometric methods for simultaneous determination of binary mixtures with severely overlapping spectra. Spectrochim Acta A Mol Biomol Spectrosc 136: 1786-1796.

29. Lotfy HM, Saleh SS, Hassan NY, Salem H (2015) Computation of geometric representation of novel spectrophotometric methods used for the analysis of minor components in pharmaceutical preparations. Spectrochim Acta A Mol Biomol Spectrosc 151: 628-643.

30. Mohamed AEI, Abdel-Maaboud IM, Salem H, Maher E (2017) Chemometrics-assisted spectrophotometric determination of certain $\beta$-lactam antibiotics combinations. Thai J Pharm Sci 31: 9-27.

31. Mostafa AA, Salem H, Hegazy M, Ali O (2013) Stability Indicating Spectrophotometric and Chemometric Methods for Determination of Buflomedil in Presence of its Acid Induced Degradation Products. Anal Chem Let 3(5,6): 342-358.

32. Hegazy MA, El-Ghobashy MR, Yehia AM, Mostafa AA (2009) Simultaneous determionation of metformin hydrochloride and pioglitazone hydrochloride in binary mixture and in their ternary mixture with pioglitazone acid degradate using spectrophotometric and chemometric methods. DTA 1(7): 339-349.

33. Nasr JJ, Ashour A, Eid M, Elbrashy A, Belal F (2015) Stability indicating spectrophotometric determination of Aceclofenac using multivariate calibration. Pharm Anal Acta 6(3): 1-6.

34. Alahmad S, Elfatary HM, Mabrouk MM, Hammad SF, Mansour FR (2018) Development and validation of chemometric spectrophotometric methods for simultaneous determination of simvastatin and nicotinic acid in binary combinations. Curr Drug Discov Technol 15(2): 149-155.

35. Martens H, Naes T (1989) Multivariate calibration: A user-friendly guide to multivariate calibration and classifi cation., John Wiley \& Sons, New York. J Chemo 4(144): 504

36. Mansilla AE, Meras ID, Salinas F (1998) Simultaneous determination of pteridines in multicomponent mixtures using derivative spectrophotometry and partial least-squares calibration. J Pharm Biomed Anal 17(8): 1325-1334.

37. Haaland DM, Thomas EV (1988) Partial least-squares methods for spectral analyses. Relation to other quantitative calibration methods and the extraction of qualitative information. Anal Chem 60(11): 11931202

38. Wagieh NE, Hegazy MA, Abdelkawy M, Abdelaleem EA (2010) Quantitative determination of oxybutynin hydrochloride by spectrophotometry, chemometry and HPTLC in presence of its degradation product and additives in different pharmaceutical dosage forms. Talanta 80(5): 2007-2015. 
39. The United States Pharmacopoeia 30, NF 25 (2007) Electronic version, Washington, DC: American Pharmaceutical Association, US.
40. ICH Harmonized Tripartite Guideline Q2 (R1) (2005) Validation of Analytical Procedures: Text and Methodology. 